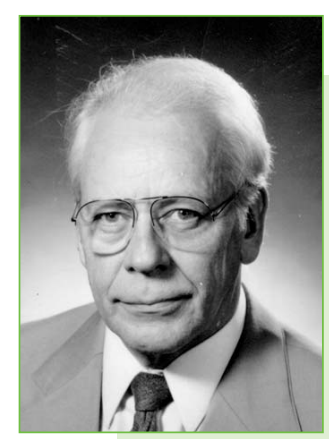

\title{
Videant consules: HMP versus NEM
}

Bemerkenswert im Zusammenhang mit dem Markt "Selbstmedikation" erscheint die Feststellung "neue Spielregeln und Spieler". So geschehen in der Ankündigung einer Vortragsveranstaltung eines großen und reputierten im Gesundheitssektor tätigen Verbandes. Der Rezensent hatte aus Zeitgründen nicht das Vergnügen "mitzuspielen" und kann sich so seine Gedanken nur zu den Überschriften dieses Events machen. Das ist natürlich auch ein gefährliches Spiel, weil hier möglicherweise pars pro toto geschlossen wird. Sei's drum! So heißt es einmal "Selbstmedikation unter veränderten Bedingungen" - gemeint sind Marktanalysen nach der bedeutenden Einschränkung einer Erstattungsfähigkeit von Arzneimitteln; hiervon sind Arzneipflanzenprodukte (HMP) überproportional betroffen. Nicht geändert dagegen haben sich hier die Forderungen nach Qualität, Sicherheit und Wirksamkeit, die Kardinaltugenden von Arzneimitteln schlechthin - und das ist gut so! Denn die Erwartungen der Verbraucher sind mit Recht hochgesteckt und dürfen nicht enttäuscht werden.

Eine andere Überschrift der gleichen Veranstaltung lautet »Neue Märkte aus Sicht der Ernährungsindustrie». Liegt hier des Pudels Kern, nämlich die Verwendung von Arzneipflanzen in Nahrungsergänzungsmitteln (NEM), vor allem aber in den »healthclaims«, den gesundheitsbezogenen Werbeaussagen solcher Mittel?

Ohne einen anderen Vertriebsweg für AM, einschl. HMP, in extenso darzustellen, wie dieser in Filialen einer Drogerie-Vertriebskette versucht wird (vgl. DÄBl 103, H.8; (Febr. 2006), gilt auch hier: Wehret den Anfängen - wohlgemerkt aus ärztlich-ethischen Erwägungen und nicht etwa aus kommerziellen Überlegungen! Diese sind dann aber sicher Sache besonderer Aufmerksamkeit der Beteiligten, wenn es gilt, einen weiteren Titel auszufüllen, nämlich: "Neue Spielregeln in der Apothekenansprache» zu suchen und zu finden. Gemeint sind offenbar strategische Fragen im Umgang mit Apotheken-Kooperationen. An sich nicht zu beanstanden, so lange die Trias Qualität, Sicherheit und Wirksamkeit durch entsprechende Untersuchungen belegt ist.

Was aber, wenn nicht? Oder wenn die Arzneipflanze im Mantel des NEM angeboten und mit semantisch geschickten »health claims» versehen dem arglosen
Editorial

Verbraucher nicht mehr unbedingt in der Apotheke verkauft wird? Erfolgt auf diese Weise eine Abwanderung von entsprechenden Arzneipflanzen in den Lebensmittelsektor, etwa als Bestandteil von Novel Food, so ist Tür und Tor offen zum Einsatz von Arzneipflanzen-Zubereitungen in Lebensmitteln, die dann neben einer Nährwertkennzeichnung auch gesundheitsbezogene Angaben enthalten. Mit Hypericum in Joghurt hat es angefangen, mit Sabal könnte es weitergehen. Als Lebensmittel, eben nur als NEM, in den Handel gebracht ohne Beeinflussungsmöglichkeit der Verzehrsmenge, d.h. ohne Kontrolle effektiver oder gar überschrittener Dosis, ohne Überprüfung der Qualität, vor allem aber ohne Hinweise auf mögliche unerwünschte Neben- oder Wechselwirkungen - diese werden nicht auf der NEM-Packung erscheinen noch bei einem Auftreten korrekt zugeordnet werden - ganz zu schweigen von einer fehlenden, weil prima vista nicht geforderten Einhaltung von Arzneibuch- oder Zulassungskriterien.

Aus diesem Dilemma könnte vielleicht eine Positivliste von Arzneipflanzen, die nicht in Lebensmitteln zu verwenden sind, herausführen. Für diese Liste sollten zeichnen in gegenseitiger Übereinkunft der Beteiligten: Vertreter der Wissenschaft in den Heilberufen, Verbraucherinitiativen und Hersteller. In dieser Liste sollten dann alle Arzneipflanzen aufgeführt werden, die entsprechend ihrer lange beobachteten Heilwirkungen untermauert mit modernen Wirksamkeitsnachweisen geeignet sind, gesicherte medizinische Indikationen zu beanspruchen.

2007 ist nicht mehr fern. Dann stehen bei der Europäischen Kommission (EC) Entscheidungen der vorberichteten Art an. Daher sollten, nein müssen, die Vertreter der Heilberufe, Ärzte und Apotheker - die Consuln - gemeinsam im Interesse der ihnen anvertrauten Patienten und Ratsuchenden gegen eine $\mathrm{Ab}$ sicht der EC zur Erweiterung der Direktive 2002/46 EG, zumindest soweit diese anerkannte Arzneipflanzen betrifft, geschlossen auftreten, ne quid res publica/salus aegroti detrimenti capiat! damit (frei übersetzt): die gute Sache der pflanzlichen Arzneimittel und deren Verwendung keinen Schaden leidet.

Fritz H. Kemper, Münster 